\title{
Pensononowoor
}

2018, vol. 79, 47-60

http://dx.doi.org/10.12657/denbio.079.005

\author{
Hooman Ravanbakhsh, Behnam Hamzeh'ee, Alireza Moshki
}

\section{Ecology and phytosociology of Cotoneaster shrublands in Central Alborz of Iran}

Received: 8 April 2017; Accepted: 31 October 2017

\begin{abstract}
The genus Cotoneaster is considered an important taxon in the woodlands of the Mediterranean and Irano-Turanian regions. Some species of this genus were reported from the Irano-Turanian alpine woodlands. Irano-Turanian mountainous wood and shrublands have a great importance in terms of water and soil conservation, biodiversity and plant richness. There is a lack of quantitative and qualitative statistics available for many of these ecosystems. This research focused on the ecology and phytosociology of Cotoneaster shrublands in central Alborz (Iran), with emphasis on C. kotschyi, an endemic drought-tolerant species. Data was collected based on the Braun-Blanquet method. TWINSPAN was used to analyse the vegetation data. Species-environment analysis was performed by CCA (Canonical Correspondence Analysis) and oneway ANOVA.

Relevés were classified into three distinct groups regarding their floristic composition. By organizing the phytosociological table, a new subassociation was defined and named as Rhamno pallasii-Juniperetum excelsae cotoneastretosum kotschyi subass. nova. This syntaxon is distributed in the range of 2,200-2,430 m a.s.1. between two other groups, i.e. Cotoneastro nummulariis-Juniperetum excelsae and Rhamno pallasii-Juniperetum excelsae. Cotoneaster kotschyi ecologically is near to Rhamnus pallasii which is characteristic for Juniper communities on shallow soils and stony lands. Among the environmental variables, slope, soil texture, $\mathrm{pH}$, lime and saturation percent are the most important distinguishing factors of this subassociation. So, the new syntaxon is found in the habitat with an average slope of $60 \%$, sandy-loam soils and $\mathrm{pH}$ and lime percent less than other studied communities. The subassociation cotoneastretosum kotschyi has a higher amount of sand content compared to the other vegetation groups.

Cotoneaster nummularius is an indicator of vegetation communities with relatively evolved soils. However, $C$. kotschyi grows in poor and shallow soils. C. kotschyi is a differential species which indicates the variability between the two main Alpine associations of the Irano-Turanian region. It is an appropriate species for plantation in the semi-arid mountainous areas. The ecological demands and the floristic composition of these plantations are determined in this article.
\end{abstract}

Keywords: Cotoneaster kotschyi, Juniperus excelsa, species-environment relationships, Mediterranean, Irano-Turanian shrublands

Addresses: H. Ravanbakhsh, A. Moshki, Department of Forestry, Faculty of Desert Studies, Semnan University, Semnan, Iran, e-mail: h.ravanbakhsh@semnan.ac.ir

B. Hamzeh'ee, Department of Botany, Research Institute of Forests and Rangelands, Agricultural Research Education and Extension Organization (AREEO), P. O. Box 13185-116, Tehran, Iran 


\section{Introduction}

Mountainous forests play an important role in terms of water and soil conservation, biodiversity, rehabilitation of vegetation and wildlife protection. These functions are more substantial in arid and semi-arid areas. The Irano-Turanian region, is one of the great phytogeography zones of arid lands in the world, and mostly consists of steppes and deserts. However, it partially covers some mountainous forests and woodlands which further make it an unique ecosystem. In this region, phanerophytes mainly consist of shrubs. Almost all of them are tolerant to harsh conditions such as drought and cold. Furthermore, shrubs are an important component of mountain ecosystems in terms of productivity and diversity (Elzein et al., 2011). The dominant species in forest communities in the mountainous regions of Irano-Turanian zone are Juniperus excelsa M.Bieb., Pistacia spp. and Amygdalus spp. (Sagheb-talebi et al., 2014). Species such as Cotoneaster spp., Berberis spp., Lonicera spp., Rhamnus pallasii Fisch. \& C.A.Mey, Cerasus microcarpa Boiss., Rosa spp. and occasionally Paliurus spina-christi Mill. occur with Juniper trees (Zohary, 1973; Klein, 2001; Kartoolinejad \& Moshki, 2014; Ravanbakhsh et al., 2016). It is believed that these forest stands and scattered Juniper trees were originally steppe forests that were likely a dominant vegetation type in all southern slopes of the Alborz Mountains (Zohary, 1973). These forest and woodland communities belong to the class Junipero-Pistacietea Zohary 1973.

Various species of the genus Cotoneaster constitute a dominant species of the Irano-Turanian shrublands. Cotoneaster kotschyi (C.K.Schneid.) G.Klotz is an endemic shrub species (Ried, 1969; Khatamsaz, 1992) that often occurs sporadically in the southern slopes of the Alborz Mountains and Kerman (Khatamsaz, 1992), but rarely appears in certain landscapes with high sociability and forms shrub communities (Ravanbakhsh et al., 2010). This taxon has not yet been assessed for the IUCN Red List (2016), but its habitat is mainly endangered by human activities. $C$. kotschyi is very tolerant to drought that makes it a suitable species for reforestation and carbon sequestration projects in arid and semi-arid mountainous regions. Ravanbakhsh et al. (2010) found C. kotschyi in South Alborz at 1,950 to 2,650 m a.s.l. along with Juniperus excelsa, Amygdalus lycioides Spach and Cerasus microcarpa. Mohammadi et al. (2015) studied the traditional use of this species and showed that Cotoneaster fruit can be used to treat asthma. Various species of Cotoneaster have the diagnostic role in phytosociology of Mediterranean and sub-Mediterranean regions. Cotoneaster nummularius Fisch. \& C.A.Mey. along with Juniperus oxycedrus L. and Berberis crataegina DC. are indicator species of shrub story in
Querco vulcanicae-Juniperetum excelsae Kargioglu 2005 in Turkish Yandag forests (Kargioglu \& Tatli, 2005). This community can be generally observed on the limestone bedrock covered by brown forest soil with slopes of $5-20 \%$ and altitude of 1,300 to $1,600 \mathrm{~m}$ a.s.l. Rhamnus pallasii and Cotoneaster nummularius grow well in the eroded areas of the Firat valley in Turkey and are suitable for preventing erosion (Kaya, 1999). The species of the genus Cotoneaster along with Prunus, Rosa and Quercus are the pioneer species in succession steps in Cedrus libani A.Rich. forests (Beals, 1965). Some species of Cotoneaster were considered as protected shrubs of the Polish Sudety Mountains (Boratyński et al., 1999). Cotoneaster nummularius and Lonicera nummulariifolia Jaub. \& Spach are characteristic species of Cotoneastro nummulariis-Juniperetum excelsae Ravanbakhsh \& Hamzeh'ee 2015 which occurs in the 2,250-2,750 $\mathrm{m}$ a.s.l. on loam, clay loam and sandy loam soils in the South Alborz (Ravanbakhsh et al., 2016). Cotoneaster racemiflora K.Koch. and Rosa laccrans Boiss. \& Buhse occur in Juniper forest communities of the Himalayas in different geographical directions from 2,100 to 2,800 $\mathrm{m}$ a.s.l. (Ahmed, 2006).

Natural resources management and sustainable development are based on initial recognition and analysis of vegetation, which provides a basis to prevent the extinction of species or plant communities. The identification and analysis of plant communities, especially forest communities, can provide an example for the rehabilitation and development of vegetation communities, particularly in arid and semi-arid regions. Therefore, the objective of this study was the analysis of vegetation and species-environment relationships in Cotoneaster shrublands of Alborz mountains, with emphasis on the ecological behaviour of C. kotschyi.

\section{Material and methods}

\section{Study area}

This study was carried out in the Central Alborz Mountains. Two species of Cotoneaster, C. nummularius and C. kotschyi, along with some other species that constitute shrublands of the Central Alborz. C. nummularius has a wide distribution in shrublands, but C. kotschyi often grows sporadically. The Rooteh Forest Reserve of Central Alborz is one of these areas, where the species are observed in shrubland formation with a high degree of sociability. Therefore, this habitat was selected for this phytosociological study (Fig. 1). This habitat with an area of 7.5 hectares was located next to the Rooteh village. The mean annual precipitation of this region is $687 \mathrm{~mm}$, mostly in the form of snow. The dry season of this area 


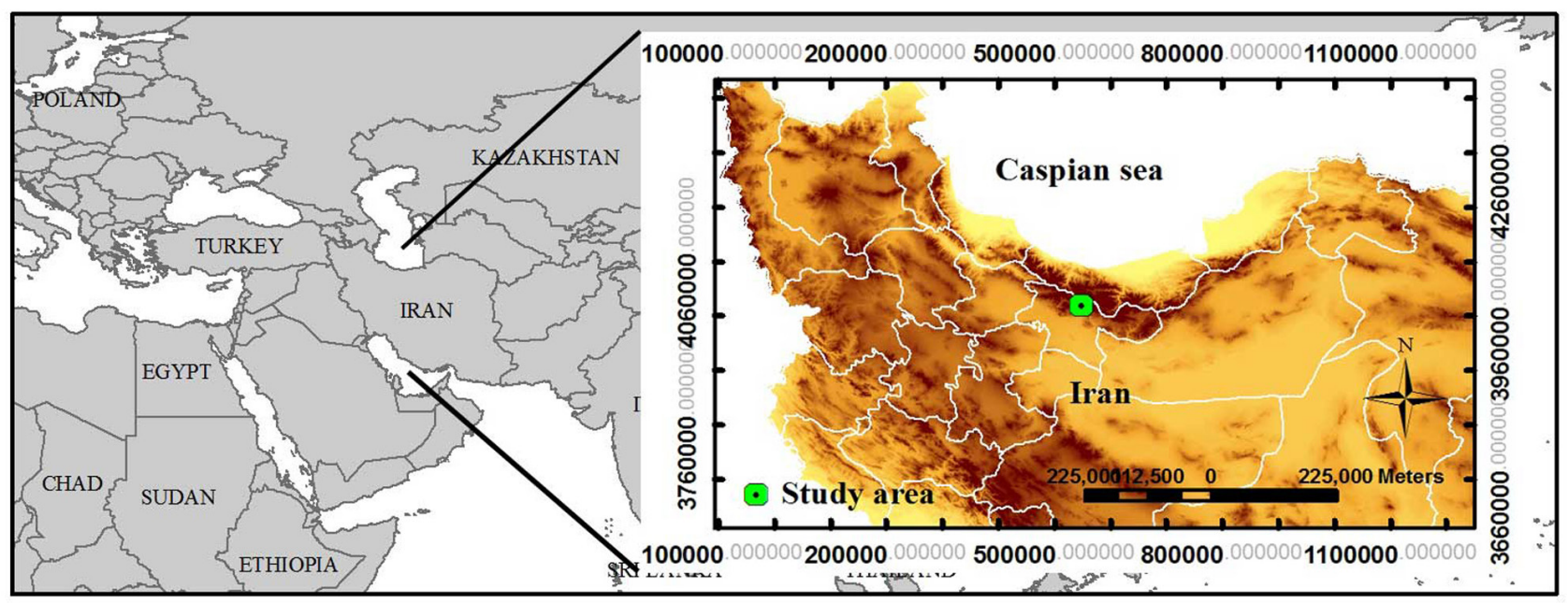

Fig. 1. Location of Cotoneaster kotschyi stand

lasts about $4-5$ months. The geological formations of sedimentary rocks consist of siltstone, shale, dolomite and conglomerate. Soils are typically antisols and inceptisols.

\section{Data collection and analysis}

Field data were collected based on the Braun-Blanquet method (Braun-Blanquet, 1951; Biondi, 2011). For each phytosociological relevé a set of environmental data including topography and soil properties were also recorded. The soil samples were taken from the $10-30 \mathrm{~cm}$ depth. In addition to the relevés belonging to $C$. kotschyi community, some relevés of related communities were considered in order to prepare the phytosociological table. A total of 27 relevés were taken. The recognition of plant species was performed using Flora of Iran (Assadi, 1988-2016) and Flora Iranica (Rechinger, 1963-2005).

The analysis of the vegetation data was performed by TWINSPAN (Hill, 1979). After sorting vegetation with TWINSPAN, the diagnostic species were determined. Diagnostic (characteristic and differential) species are species with the distinct concentrations of occurrence or abundance in a particular vegetation unit (Chytrý \& Tichý, 2003). The diagnostic species can be used as characteristic species to diagnose plant associations, or for determining the subassociation as differential species. The diagnostic value of species was based on the fidelity concept, which was considered as dependence of one special species within a particular community (Poore, 1955). To calculate fidelity the Chytrý et al. (2002) method was applied using JUICE ver. 7.0 software (Tichý, 2002). Using Fisher's exact test, the significance of fidelity values were investigated at the $1 \% \mathrm{P}$-value (Tichý, 2002). The diagnostic species were controlled and confirmed based on their chorology, viability, ecological properties and bibliography. Afterwards, characteristic, differential and companion species were determined. The nomenclature of new syntaxon was applied according to the International Code of Phytosociological Nomenclature, $3^{\text {rd }}$ edition (Weber et al., 2000).

The ordination method was used to assess the species-environment relationships (Kent \& Coker, 1994). Since the species data in this study generally showed a non-linear species response curve, CCA (Canonical Correspondence Analysis) was applied to investigate the vegetation-environment relationships (Lepš \& Šmilauer, 1999) using PC-ORD 4 software (McCune \& Mefford, 1999) and Canoco 4.5 (ter Braak \& Smilauer, 2002). For the application of ordination method, the different measurement units of environmental variables were standardized (ter Braak, 1986). The significance of the CCA axes and species-environment correlations were assessed using the Monte-Carlo test.

In multivariate analysis, the environmental variables should not be a linear combination of variables. This problem can occur for example in the case of soil texture parameters (sand, silt and clay), which entails the removal of one of variables in each case (Palmer, 1993). Therefore, here the variable silt was removed from CCA ordination. The variable aspect was investigated based on the four main directions (90, 180, 270 and 360) and was applied in the analysis after being categorized into four classes of artificial variables. This results in an easier interpretation of data (Palmer, 1993). Due to the large number of species and relevés, their presentation in a single diagram was impossible. Therefore, highly-correlated species were used for presentation in the diagram. Furthermore, the ANOVA followed by Duncan analysis was used to compare the effects of environmental parameters (i.e. soil and topographic variables) in different vegetation groups using IBM SPSS Statistics ver. 22. 


\section{Results}

\section{Species classification}

The relevés were classified into three distinct groups regarding the floristic composition using TWINSPAN method. The first and second groups showed similar floristic compositions and placed with each other in a larger group. In the following, the groups will be explained based on the results presented by Juice software.

1. Group 1

- Constant species: Juniperus excelsa, Rhamnus pallasii

- Diagnostic species: Rhamnus pallasii, Ephedra major Host

2. Group 2

- Constant species: Rhamnus pallasii, Cotoneaster kotschyi, Cerasus microcarpa, Amygdalus lycioides

- Diagnostic species: Cotoneaster kotschyi, Valerianella tuberculata Boiss., Gundelia tournefortii L., Amygdalus lycioides, Pistacia atlantica Desf.

3. Group 3

- Constant species: Juniperus excelsa, Cotoneaster nummularius, Lonicera nummulariifolia, Berberis integerrima Bunge., Hypericum scabrum L., Dactylis glomerata L.

- Diagnostic species: Cotoneaster nummularius, Lonicera nummulariifolia, Astragalus aegobromus Boiss. \& Hohen., Cousinia calocephala Jaub. \& Spach.

\section{Phytosociology}

Following the classification of vegetation groups, the relevés and species were arranged in phytosociological table (Table 1). The analysis of the characteristic species of each group and comparing them with published syntaxa (Table 2) showed that the first and third groups can be classified as Rhamno pallasii-Juniperetum excelsae and Cotoneastro nummulariis-Juniperetum excelsae (Tables $1 \& 2$ ), whereas the second group is a new syntaxon. Therefore the bibliography for floristic composition of the new syntaxon (with emphasis on Rhamnus pallasii, Cotoneaster kotschyi, Valerianella tuberculata and Pistacia atlantica) is provided and organized in the synoptic table (Table 2). The species Pistacia atlantica is recognized as a diagnostic species for the new syntaxon. This species was already listed as characteristic species of other associations (Zohary, 1973; Quézel et al., 1980; Togonidze, 2011) (Table 2). In addition, $P$. atlantica appears in the altitude of 1,300$1,800 \mathrm{~m}$ a.s.l. (Marvie Mohadjer, 2005), whereas its presence in our study area was not in the typical altitude of it. Therefore, the presence of $P$. atlantica along with the characteristic species of Rhamno pallasii-Juniperetum excelsae, as well as an endemic species
C. kotschyi represented their differential role to establish a new subassociation. This floristic composition is unique and has never been described yet (Table 2). Based on the synoptic table, the presence of Rhamnus pallasii, Cerasus microcarpa, Berberis integerrima and Conringia planisiliqua Fisch. \& C.A.May. in the floristic composition, as the characteristic species of order Juniperetalia excelsae Ravanbakhsh \& Hamzeh'ee 2015, and Juniperus excelsa and Amygdalus lycioides, as the characteristic species of class Junipero-Pistacietea Zohary 1973, indicates that this new syntaxon belongs to these order and class. Juniperus excelsa, Rhamnus pallasii and Pistacia atlantica were reported as characteristic species of some other associations in the Mediterranean and Caucasus (Table 2), but most of them belong to Quercetea pubescentis Doingt \& Kraft 1955 (Tel et al., 2010) and their floristic composition is considerably different from the Alborz associations.

Therefore, the classification of the new syntaxon and the related syntaxa are as follow:

- Class: Junipero-Pistacietea Zohary 1973

- Order: Juniperetalia excelsae Ravanbakhsh \& Hamzeh'ee 2015

- Association: Rhamno pallasii-Juniperetum excelsae Ravanbakhsh \& Hamzeh'ee 2015

- Subassociation:

- Rhamno pallasii-Juniperetum excelsae cotoneastretosum kotschyi subass. nova hoc loco

- Holotypus: Table 1, rel. 25

- Differential species: Cotoneaster kotschyi, Valerianella tuberculata, Pistacia atlantica

- Higher syntaxa characteristic species: Juniperus excelsa, Amygdalus lycioides, Rhamnus pallasii, Cerasus microcarpa, Berberis integerrima, Conringia planisiliqua, Ephedra major

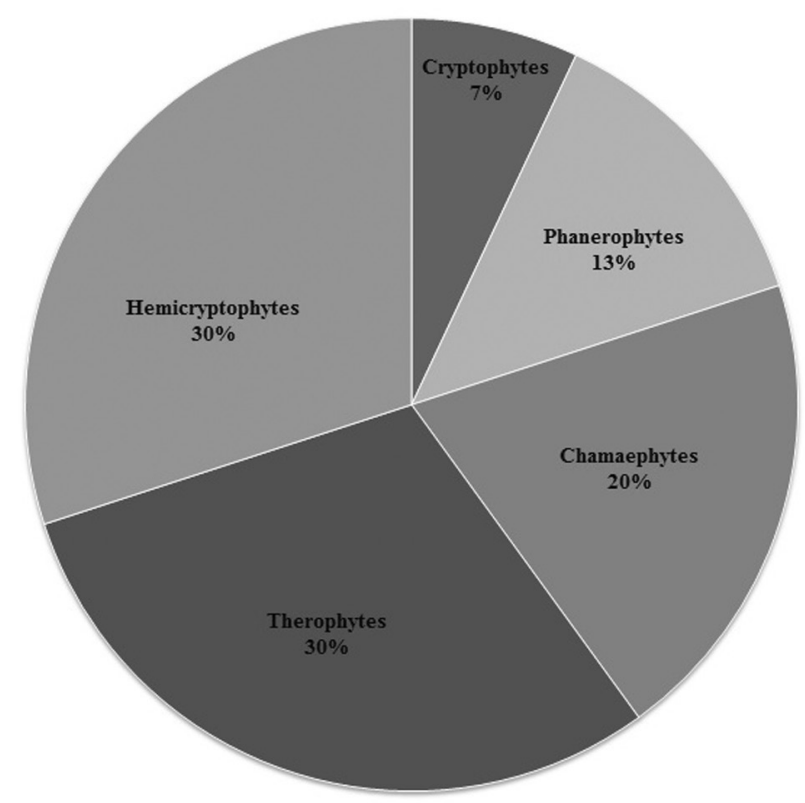

Fig. 2. Life form groups in Cotoneaster kotschyi habitat 
Table 1. Floristic composition in the studied communities (Col. 1-16: Rhamno pallasii-Juniperetum excelsae; Col. 1-6: Rp-Je cotoneastretosum kotschyi; Col. 17-26: Cotoneastro nummulariis-Juniperetum excelsae)

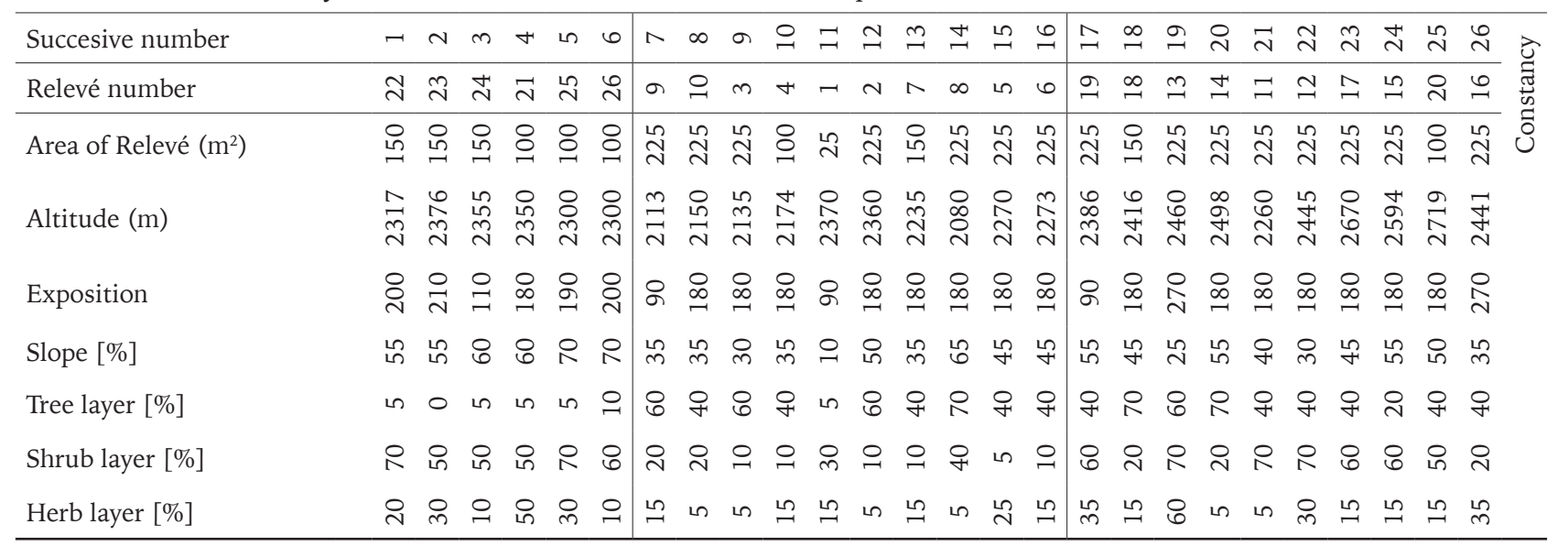

I. ChAss. Rhamno pallasii-Juniperetum excelsae

Rhamnus pallasii $\quad \mathrm{b} \quad$\begin{tabular}{llllllllllllllll|}
2 & 1 & 3 & 3 & 2 & 2 & 2 & 3 & 3 & 3 & 3 & 2 & 2 & 3 & 2 & 3 \\
\hline
\end{tabular} IV

II. DSubass. cotoneastretosum kotschyi

$\begin{array}{llllllll}\text { Cotoneaster kotschyi } & \mathrm{b} & 3 & 3 & 2 & 3 & 4 & 3 \\ \text { Valerianella tuberculata } & & + & + & . & 1 & 1 & 1 \\ \text { Pistacia atlantica } & \mathrm{a} & + & . & . & . & 1 & +\end{array}$

III. ChAss. Cotoneastro nummulariis-Juniperetum excelsae

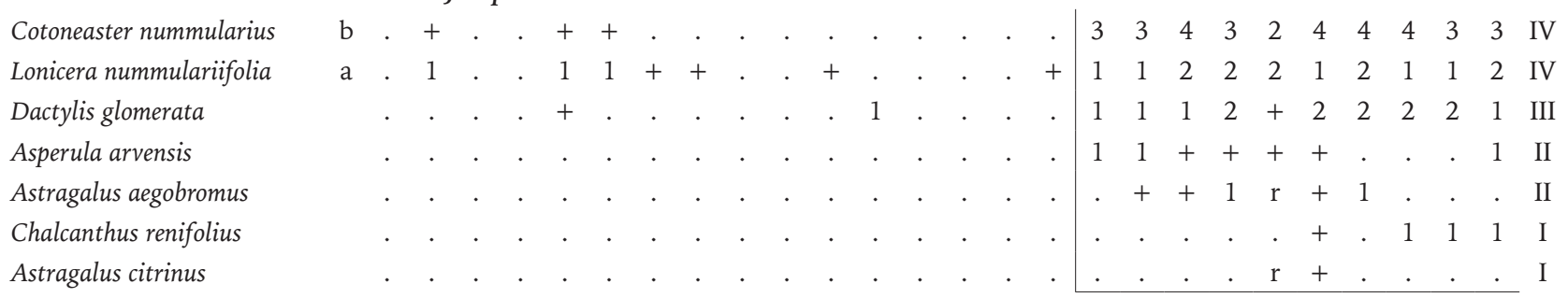

IV. ChO. Juniperetalia excelsae \& ChCl. Junipero-Pistacietea

\begin{tabular}{|c|c|c|c|c|c|c|c|c|c|c|c|c|c|c|c|c|c|c|c|c|c|c|c|c|c|c|c|c|}
\hline Juniperus excelsa & $\mathrm{a}$ & . & + & . & 1 & . & 2 & 4 & 4 & 4 & 3 & + & 3 & 4 & 4 & 4 & 4 & 3 & 4 & 4 & 4 & 3 & 4 & 3 & 3 & 4 & 3 & V \\
\hline Berberis integerrima & $\mathrm{b}$ & . & + & . & . & . & . & . & + & . & + & + & + & 1 & 2 & 1 & . & 1 & + & 2 & + & 3 & 1 & 2 & 1 & 1 & 1 & IV \\
\hline Cerasus microcarpa & $\mathrm{b}$ & 1 & 1 & 1 & 1 & 2 & 1 & . & $\cdot$ & . & . & . & . & . & + & . & + & ${ }^{\circ}$ & 1 & 3 & + & 3 & 2 & 1 & 1 & + & 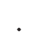 & IV \\
\hline Rubia florida & & . & . & . & . & . & . & 1 & . & . & 1 & $\cdot$ & . & 1 & 1 & 1 & + & 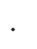 & 1 & + & 1 & 1 & + & 1 & 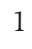 & & 1 & III \\
\hline Conringia planisiliqua & & + & + & . & . & . & . & 1 & . & + & 1 & . & 1 & + & + & - & . & + & . & $\cdot$ & 1 & . & . & . & & & 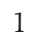 & III \\
\hline Cousinia calocephala & & . & . & . & . & . & . & 1 & $\cdot$ & $\cdot$ & . & . & $T$ & $\cdot$ & . & . & . & 1 & . & + & 1 & + & $\mathrm{r}$ & + & & & & II \\
\hline Amygdalus lycioides & $\mathrm{b}$ & 1 & 2 & 1 & 3 & + & 1 & . & . & . & + & . & + & . & + & . & . & . & . & . & . & . & . & . & . & 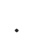 & . & II \\
\hline Berberis crataegina & $\mathrm{b}$ & . & . & . & . & . & . & 1 & . & . & . & . & $\cdot$ & . & 1 & . & . & . & . & 2 & $\cdot$ & . & 2 & . & . & . & . & I \\
\hline Ephedra major & $\mathrm{b}$ & . & . & . & . & . & . & 2 & 2 & 2 & 2 & . & . & 1 & 2 & . & 2 & . & ${ }^{\circ}$ & . & $\cdot$ & . & . & . & . & $\cdot$ & . & II \\
\hline Rosa canina & $\mathrm{b}$ & + & . & + & . & . & . & $\cdot$ & $\cdot$ & . & . & . & . & $\cdot$ & . & . & . & . & . & $\cdot$ & $\cdot$ & . & 2 & . & + & . & . & I \\
\hline Silene aucheriana & & . & . & . & + & . & . & $\cdot$ & $\cdot$ & . & . & . & . & . & . & . & . & . & . & + & . & + & $\cdot$ & . & . & 1 & . & I \\
\hline Silene marschallii & & . & . & . & . & . & . & $\cdot$ & $\cdot$ & . & . & . & . & . & . & $\mathrm{r}$ & . & . & ${ }^{\circ}$ & $\cdot$ & $\cdot$ & . & + & . & . & . & + & I \\
\hline Celtis caucasica & $\mathrm{a}$ & + & . & . & . & . & $\cdot$ & . & $\cdot$ & . & $\cdot$ & . & $\cdot$ & $\cdot$ & . & $\cdot$ & $\cdot$ & . & . & $\cdot$ & . & . & . & . & . & . & . & I \\
\hline \multicolumn{29}{|l|}{ V. Others } \\
\hline Psathyrostachys fragilis & & + & . & 1 & + & + & 1 & 1 & 1 & 1 & + & . & . & + & 1 & 1 & 1 & 1 & 1 & . & . & 1 & . & + & 5 & . & + & IV \\
\hline Verbascum speciosum & & + & + & + & + & + & 1 & $\mathrm{r}$ & + & + & . & + & . & + & + & + & . & + & 1 & . & + & + & + & . & + & r. & . & IV \\
\hline Euphorbia cheiradenia & & + & . & + & . & + & . & 1 & 1 & 1 & + & + & . & 1 & 1 & . & . & 1 & + & + & + & 1 & 1 & 1 & + & 1 & . & IV \\
\hline Alyssum minus & & 1 & + & + & 1 & + & 1 & 1 & + & 1 & 1 & 1 & 1 & 1 & 1 & 1 & 1 & . & 1 & 1 & 1 & 1 & 1 & 1 & & & 1 & $\mathrm{~V}$ \\
\hline Acinos graveolens & & 1 & 1 & + & 1 & 1 & 1 & . & 1 & $\cdot$ & . & + & 1 & $\cdot$ & 1 & 1 & 1 & . & 1 & 1 & + & + & + & . & 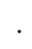 & . & . & IV \\
\hline Senecio vernalis & & 1 & 1 & 1 & 1 & 1 & 1 & 1 & $\mathrm{R}$ & 1 & 1 & 1 & . & 1 & . & + & . & 1 & 1 & + & 1 & . & 1 & 1 & + & . & . & IV \\
\hline Bromus tectorum & & 1 & 1 & 1 & 1 & 1 & 1 & . & 1 & 1 & 1 & 1 & $\cdot$ & 1 & + & 1 & 1 & 1 & 1 & 1 & 1 & + & 1 & 1 & & • & . & $\mathrm{V}$ \\
\hline Astragalus verus & & 1 & 1 & 1 & 1 & . & 1 & . & . & . & + & + & 2 & . & . & + & + & + & . & 1 & + & 1 & + & . & & & + & IV \\
\hline Hypericum scabrum & & + & . & . & . & . & + & 1 & . & . & $\cdot$ & . & . & . & . & $\cdot$ & . & + & 1 & 1 & 1 & + & 2 & 2 & 2 & 1 & 2 & III \\
\hline Geranium persicum & & 1 & + & 1 & 1 & 1 & 1 & . & . & . & + & 1 & 1 & . & . & + & . & . & . & + & 1 & . & + & + & • & + & . & III \\
\hline
\end{tabular}




\begin{tabular}{|c|c|c|c|c|c|c|c|c|c|c|c|c|c|c|c|c|c|c|c|c|c|c|c|c|c|c|c|}
\hline Succesiv & $r$ & N & $n$ & + & in & 6 & $\wedge$ & $\infty$ & $\sigma$ & $\stackrel{\circ}{\longrightarrow}$ & $\exists$ & 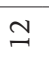 & $\stackrel{m}{\sim}$ & $\stackrel{\leftrightarrows}{\leftrightarrows}$ & $\stackrel{n}{=}$ & $\stackrel{0}{\sim}$ & 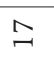 & $\stackrel{\infty}{\sim}$ & $\stackrel{\theta}{-}$ & 이 & $\vec{N}$ & ส & $\stackrel{\sim}{\sim}$ & $\stackrel{\leftrightarrow}{\sim}$ & $\stackrel{\llcorner}{\sim}$ & $\stackrel{\sim}{\sim}$ & \\
\hline Relevé number & ป & $\stackrel{\sim}{\sim}$ & $\stackrel{\leftrightarrow}{\sim}$ & $\vec{\lambda}$ & $\stackrel{\Perp}{\sim}$ & $\stackrel{\sim}{\sim}$ & $a$ & $\stackrel{ }{\circ}$ & $m$ & r & $\rightarrow$ & N & $\wedge$ & $\infty$ & in & 0 & $\stackrel{\sim}{\circ}$ & $\stackrel{\infty}{\sim}$ & $\stackrel{m}{\sim}$ & 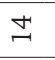 & $\exists$ & $\approx$ & $\beth$ & $\stackrel{2}{\sim}$ & 오 & $\because$ & \\
\hline Lamin & 1 & r & 1 & 1 & 1 & 1 & & 1 & & 1 & & 1 & & 1 & 1 & 1 & & . & . & . & . & + & & & 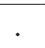 & $\cdot$ & \\
\hline Arabis nova & 1 & 1 & . & 1 & 1 & 1 & & . & 1 & 1 & & 1 & & 1 & 1 & 1 & 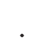 & . & . & . & . & + & . & 1 & 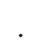 & + & \\
\hline Galium aparine & 1 & 1 & + & 1 & 1 & 1 & . & . & 1 & 1 & & . & 1 & 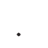 & 1 & 1 & . & 1 & 1 & 1 & 1 & 1 & 1 & 1 & . & . & V \\
\hline Lappula sinaica & + & . & + & + & + & 1 & . & . & + & + & . & + & + & . & 1 & 1 & + & . & . & . & . & + & . & . & . & +1 & . \\
\hline ubium & + & . & + & + & + & 1 & 1 & . & . & + & 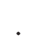 & . & . & + & + & + & . & . & . & . & . & . & . & . & . & . & \\
\hline Arte & . & . & . & . & . & . & 1 & 1 & + & + & . & + & 1 & 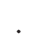 & . & + & 1 & 1 & + & . & + & + & . & 1 & 1 & 1 & Il \\
\hline Eremurus sp & . & . & . & . & . & . & . & . & + & . & 1 & 1 & + & . & + & + & + & 1 & 1 & + & 1 & 1 & 1 & + & . & 1 & II \\
\hline C & . & 1 & . & . & . & 1 & 1 & . & . & . & . & . & 1 & 1 & 1 & 1 & 1 & 1 & . & 1 & 1 & . & 1 & . & 1 & 1 & \\
\hline Alys & . & + & . & 1 & + & . & . & 1 & . & 1 & . & . & 1 & . & 1 & 1 & 1 & . & 1 & 1 & . & 1 & . & . & . & . & Il \\
\hline Sca & + & . & + & 1 & 1 & . & . & . & . & . & . & 1 & 1 & . & + & . & 1 & . & + & 1 & + & 1 & 1 & . & . & . & Il \\
\hline & . & + & . & . & 1 & 1 & . & + & + & + & 1 & . & 1 & . & . & + & 1 & . & 1 & . & + & . & . & . & . & . & \\
\hline Ver & + & + & . & . & . & . & . & . & . & . & . & 1 & . & . & . & . & 1 & 1 & 1 & 1 & . & 1 & 1 & 1 & . & +1 & III \\
\hline Eremopoa persica & . & . & . & 1 & 1 & 1 & 1 & 1 & + & 1 & 1 & 1 & 1 & 1 & 1 & 1 & 1 & . & 1 & . & . & . & . & . & . & + & II \\
\hline Ast & . & + & . & 1 & . & . & . & . & . & . & . & . & . & . & . & . & 1 & . & + & . & . & . & + & + & 1 & . & \\
\hline Call & + & + & . & 1 & 1 & . & . & . & . & . & . & . & . & . & 1 & . & . & . & + & . & + & + & . & . & . & . & 1 \\
\hline Ziziphora tenuior & 1 & 1 & + & 1 & 1 & + & + & . & + & 1 & . & . & 1 & . & . & . & . & . & . & . & . & . & . & . & . & . & II \\
\hline Tuli & + & . & + & 1 & 1 & 1 & . & . & . & + & . & 1 & $\mathrm{r}$ & . & + & . & . & . & . & . & . & . & . & . & . & . & I \\
\hline Aca & . & . & . & . & . & . & 2 & 1 & + & + & . & . & 1 & . & . & 1 & . & . & . & . & . & . & . & . & . & + & 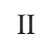 \\
\hline Onobrychis cor & . & . & . & . & . & . & 1 & . & . & . & + & . & . & + & . & . & . & 1 & 1 & 1 & . & . & 1 & 2 & . & + & II \\
\hline$S t i$ & . & . & . & . & . & . & . & + & . & + & + & + & + & . & . & + & . & . & . & . & . & . & . & . & 1 & . & \\
\hline$E l$ & 1 & . & . & . & + & . & . & . & . & . & . & . & . & . & 1 & . & 1 & 1 & . & . & 1 & . & 1 & . & . & . & I \\
\hline Meli & . & . & . & . & . & . & . & . & . & . & . & . & . & . & . & . & 1 & . & + & . & + & $\mathrm{r}$ & + & + & . & . & I \\
\hline Alli & . & + & . & 1 & 1 & 1 & . & . & . & . & . & . & . & . & . & . & . & . & . & . & . & + & 1 & . & . & . & II \\
\hline $\mathrm{Hol}$ & . &. & . & . & . & . & . & . & . & . & . & . & 1 & + & 1 & + & . & . & . & . & + & + & . & . & ${ }^{\circ}$ & 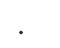 & \\
\hline Clyped & + &. & + & + & + & . & . & . & . & . & . & . & + & . & 1 & . & . & . & . & . & . & . & . & . & . & . & I \\
\hline Viol & . &. & . & . & . & . & . & . & . & 1 & + & . & 1 & . & + & + & 1 & . & . & 1 & + & 1 & . & . & 1 & . & II \\
\hline Des & . &. & . & . & . & . & 1 & . & + & . & + & . & + & . & + & $\mathrm{r}$ & . & . & . & . & . & . & . & . & . & 1 & I \\
\hline Cerato &. &. & . & . & . & . & 1 & 1 & + & + & + & . & 1 & . & . & . & . & . & . & . & . & . & . & . & 1 & . & I \\
\hline Veron & . & . & . & . & . & . & 1 & . & + & 1 & . & . & + & 1 & 1 & 1 & . & . & . & . & . & . & . & . & . & . & I \\
\hline $\mathrm{Cre}$ & . & + & . & . & . & + & . & . & . & . & + & . & . & . & $\mathrm{r}$ & . & . & . & . & . & + & . & + & . & . & . & I \\
\hline Scan & . & + & . & + & + & 1 & . & . & . & . & . & 1 & . & . & 1 & . & . & . & . & . & . & . & . & . & . & . & \\
\hline Scandix aucheri & . & . & . & . & . & . & . & . & . & . & . & . & 1 & . & . & . & + & . & 1 & . & . & 1 & . & . & . & . & I \\
\hline Centa & . & + & + & + & . & . & . & . & . & . & . & . & . & . & . & . & + & . & . & . & + & + & 1 & 1 & . & . & \\
\hline Teucrium polium & . & + & 1 & . & . & + & 1 & 1 & 1 & . & . & . & 1 & 1 & 2 & . & . & . & . & . & . & . & . & . & . & . & \\
\hline Eryngium billardier & . & . & . & . & . & . & . & . & . & . & . & + & . & . & . & + & . & + & + & . & + & + & . & + & 1 & . & I \\
\hline Alyssum inflatum & . & . & . & . & . & . & + & + & . & . & 1 & 1 & . & 1 & . & . & . & . & . & 1 & . & . & . & . & 1 & - & \\
\hline Stachys lavandulifo & . & 1 & 1 & . & . & 1 & . & . & 1 & . & . & . & . & . & . & . & . & . & . & . & . & . & . & . & . & . & \\
\hline Valerianella szowitsiana & . & + & . & + & . & . & . & . & . & . & . & . & . & . & . & . & . & . & . & . & . & . & . & . & . & . & I \\
\hline Acanthophyllum glan & . & . & . & . & . & . & . & . & . & . & . & . & . & . & . & . & 1 & . & + & . & + & + & . & . & . & + & \\
\hline Bromus tomentellus & . & . & . & . & . & + & + & . & . & . & . & . & + & . & . & . & . & . & . & . & . & . & . & . & 1 & 1 & \\
\hline Fumaria asepala & . & + & . & + & . & 1 & . & . & . & . & . & . & . & . & + & . & . & . & . & . & . & . & . & . & . & . & I \\
\hline Stachys inflata & 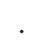 & . & . & . & . & . & 1 & 1 & . & . & . & . & . & 1 & + & . & . & . & . & . & + & . & . & . & . & . & I \\
\hline Colchicum specios & + & . & + & . & . & . & . & . & . & $\cdot$ & . & . & . & . & $\mathrm{r}$ & . & . & . & . & . & . & . & . & . & . & . & \\
\hline Allium iranicum & . & + & . & + & . & . & . & . & . & - & . & . & . & . & . & . & . & + & . & . & . & . & 1 & . & . & . & \\
\hline Marrubium vulga & . & . & . & . & . & + & . & . & . & . & ${ }^{\circ}$ & . & . & . & . & . & . & . & + & . & . & + & . & . & . & . & I \\
\hline Salvia limbata & . & . & . & . & . & + & . & . & . & - & . & . & . & . & . & . & . & 1 & + & $\cdot$ & + & . & 1 & . & . & . & I \\
\hline Muscari caucasicum & . & . & . & . & . & . & . & . & . & & 1 & & & . & . & & . & . & + & + & + & $\mathrm{r}$ & . & & 1 & . & \\
\hline
\end{tabular}


Sporadic species: I. Acanthophyllum microcephalum 14(2), Agropyron cristatum 12(+), Allium derderianum 16(+), Arenaria polycnemifolia $7(1) \& 8(+)$, Arenaria serpyllifolia 11(1), Boissiera squarrosa $14(+)$, Buglossoides arvensis $10(+) \& 11(+)$, Eremopyrum bonaepartis $8(+)$ \& 9(+), Eremopyrum confusum 14(r), Gagea reticulata 11(r), Helichrysum oligocephalum 4(+) \& 11(+), Hypericum helianthemoides 14(+), Iris pseudocaucasica 15(r), Lepyrodiclis stellarioides 13(+), Linaria lineolata 14(r)., Nepeta pungens 16(+), Polygonum molliaeforme 7(1), Rochelia persica 11(+) \&12(+), Stelleropsis iranica 7(1) \& 8(1), Thymus fedtschenkoi 15(1), Trigonella sp. 11(+), Valantia sp. 10(+), 11(+) \& 16(+), Valerianella plagiostephana $9(+) \& 13(+)$, Verbena officinalis $5(+) \& 10(+)$, Veronica rubrifolia $15(+) \& 16(+)$, Vicia vernalosa 14(+), Ziziphora clinopodioides 11(+) \& 3(1). II. Asyneuma amplexicaule 1(+), Gundelia tournefortii 2(1) \& 3(1), Isatis cappadocica 2(+) \& 3(1), Linaria simplex 4(+), Malcolmia africana 4(+), Parietaria judaica 1(+) \&4(1), Phlomis olivieri 6(+), Sanguisorba minor 5(+), Sisymbrium irio 4(+), Tanacetum parthenium 4(r), Tragopogon sp. 1(+). III. Acantholimon erinaceum 26(r), Aethionema arabicum 19(+), Aethionema cordatum 23(1) \& 26(1), Cardaria draba 22(1), 24(1) \& 25(1), Chaerophyllum macropodum 19(r), Cirsium congestum 22(+) \& 23(r), Cirsium strigosum 17(+), 23(+) \& 25(1), Convolvulus arvensis 23(+), Galium mite 23(r), Herniaria incana 26(+), Lappula barbata 23(1), Mesostomma kotschyanum 23(+), Minuartia lineata 22(+), Oryzopsis holciformis 22(r), Rosa beggeriana 21(1) \& 22(+), Rosa persica $21(+)$, Saponaria viscosa 24(r), Silene swertiifolia 23(+), Sisymbrium gaubae 22(1), Taeniatherum crinitum 17(+), 19(+) \& 20(1), Thesium kotschyanum 25(1), V. Alkanna bracteosa 5(+) \& 23(+), Bromus danthoniae 7(1), 14(+), 15(+), 16(+) \& 21(1), Bupleurum exaltatum $6(+), 13(+) \& 23(+)$, Cerastium dichotomum 4(+), 10(+), 11(+) \& 12(+), Cerastium inflatum 4(+), 15(r) \&17(1), Drabopsis verna $9(+), 11(+), 14(+) \& 21(1)$, Ferula ovina $1(+), 23(+) \& 25(1)$., Linaria striatella $10(+) \& 25(+)$, Nonea pulla $6(+) \& 17(+)$, Poa bulbosa 11(1), 12(1), 15(+) \& 16(+), Thlaspi perfoliatum 2(+), 5(+) \& 17(1).

Ecological conditions of the new syntaxon: This sub-association could be observed in mountainous habitat with shallow soils and rocky protrusions located on south, southwest and southeast aspects. It is distributed in 2,000-2,400 $\mathrm{m}$ a.s.l. Soil $\mathrm{pH}$ values vary between 7 and 7.6 , lime $2.5-8.5 \%$, organic matter $1-3 \%$, and in sandy loam soil textures.

In total, 98 species were identified in C. kotschyi habitat, which belong to 87 genera and 33 families. The families with the most species were: Apiaceae, Asteraceae, Lamiaceae, Rosaceae and Poaceae with $14,11,11,7$ and 6 species, respectively. The most abundant life forms were related to cryptophytes and therophytes (Fig. 2).

\section{Analysis of vegetation in relation to environmental variables}

The results of CCA analysis followed by the Monte Carlo test show that the first three components could be used for interpreting the results (Table 3). These components explain a total of $57.2 \%$ of variance in the species-environment relationships. The first component shows highly positive correlations with altitude, soil organic carbon and nitrogen (Table 4). Therefore, the first axis represents a gradient of these three variables. The second component shows a highly positive correlation with slope and sand, while it is negatively correlated with soil lime and $\mathrm{pH}$ (Table 4). In other words, moving on the positive direction of second axis, the slope increases as the soil becomes sandier. The first and second components have the highest eigenvalues, used for presenting the results in the diagram (Fig. 3).

The position of relevés (plots) in each diagram shows effective indicator species and environmental factors. The arrows represent the environmental variables gradient. The effective variables have a longer arrow. The relevés of cotoneastretosum kotschyi placed in the top left corner of the diagram (Figure 3 ), which on the one hand represents a higher slope and sandier soil and on the other hand shows lower soil $\mathrm{pH}$ and lime percentage of habitat. The position of relevés of cotoneastretosum kotschyi along the height vector indicates that this subassociation is placed between two other communities (i.e. Cotoneastro nummulariis-Juniperetum excelsae and Rhamno pallasii-Juniperetum excelsae).

The soil organic matter and nitrogen content are distinctive factors between $C$. nummulariis-J. excelsae and Rh. pallasii-J. excelsae. Rh. pallasii-J. excelsae and its subassociation cotoneastretosum kotschyi occur in the soils with lower organic matter and nitrogen compared to C. nummulariis-J. excelsae. The main difference of subassociation habitat with its above-rank syntaxon (i.e. Rh. pallasii-J. excelsae) is in soil texture, $\mathrm{pH}$, lime and slope, so that the cotoneastretosum kotschyi could be observed in the soils with a lower $\mathrm{pH}$, lower lime content, lighter soil texture (sandier) and steeper slopes.

The species location on the CCA biplots represents the characteristic and differential species of vegetation groups (Fig. 3). Cotoneaster kotschyi, Valerianella tuberculata and Pistacia atlantica are the differential species of cotoneastretosum kotschyi placed in the upper left corner of the diagram. These species show a preference for sandy soils and steep slopes. It was also observed that this species composition grows in the soils with lower $\mathrm{pH}$ values and lower lime contents compared to other vegetation groups studied here.

Cotoneaster nummularius, Lonicera nummulariifolia, Astragalus aegobromus and Cousinia calocephala were characteristic species of Cotoneastro nummulariis-Juniperetum excelsae which were located along the first axis in the positive direction matched with the location of the most relevés of the community. Species located in this area of the diagram had a preference for higher organic matter and nitrogen soil content which also tend to appear at higher altitudes in comparison to other species. In the negative direction of first axis, Rhamnus pallasii appears, suggesting that this species occurs at lower altitudes and in the soils with lower 
Table 2. Abbreviated synoptic table of 11 syntaxa in Iran, Mediterranean and Caucasus. Only species with higher constancy are shown ${ }^{1}$

\begin{tabular}{|c|c|c|c|c|c|c|c|c|c|c|c|}
\hline Column No. & 1 & 2 & 3 & 4 & 5 & 6 & 7 & 8 & 9 & 10 & 11 \\
\hline Class & \multicolumn{5}{|c|}{ Junipero-Pistacietea Zohary 1973} & $\begin{array}{l}\text { Ephed- } \\
\text { retea }\end{array}$ & \multicolumn{2}{|c|}{ Not defined } & \multicolumn{3}{|c|}{$\begin{array}{l}\text { Quercetea pubescentis Kraft } 1955 \\
\text { (Tel et al., 2010) }\end{array}$} \\
\hline . & \multicolumn{4}{|c|}{$\begin{array}{l}\text { Juniperetalia excelsae Ravanbakhsh \& } \\
\text { Hamzeh'ee } 2015\end{array}$} & 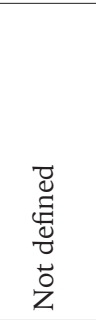 & 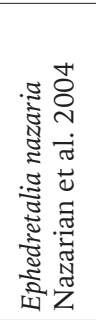 & 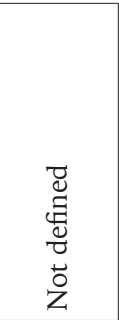 & 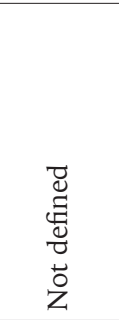 & 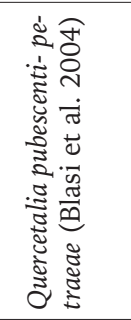 & 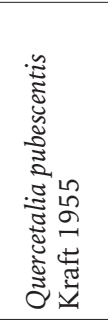 & 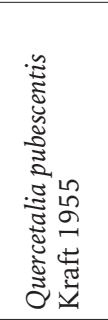 \\
\hline & \multirow{2}{*}{ 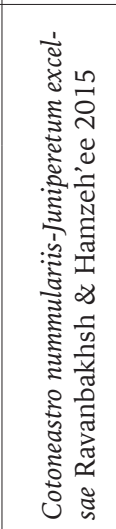 } & \multicolumn{3}{|c|}{$\begin{array}{l}\text { Rhamno pallasii-Junipere- } \\
\text { tum excelsae } \\
\text { Ravanbakhsh \& } \\
\text { Hamzeh'ee } 2015\end{array}$} & \multirow[b]{2}{*}{ 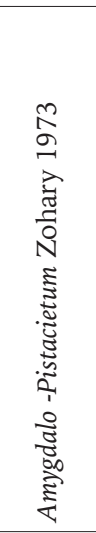 } & \multirow{2}{*}{ 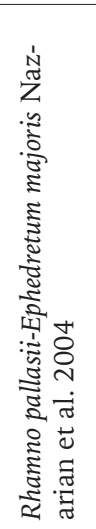 } & \multirow{2}{*}{ 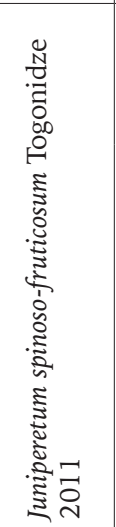 } & \multirow{2}{*}{ 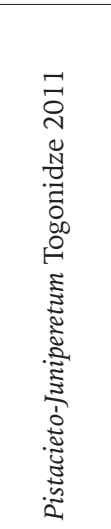 } & \multirow{2}{*}{ 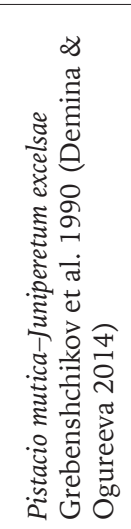 } & \multirow[b]{2}{*}{ 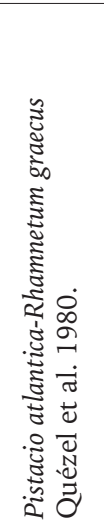 } & \multirow{2}{*}{ 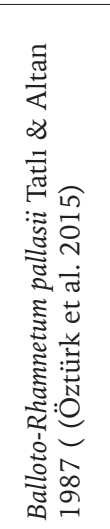 } \\
\hline $\begin{array}{l}\text { Association \& } \\
\text { Subassociation }\end{array}$ & & 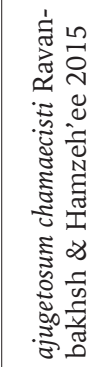 & 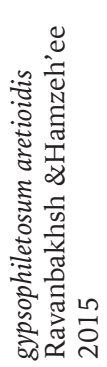 & 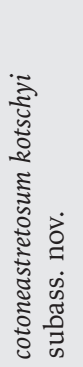 & & & & & & & \\
\hline Location & Iran & Iran & Iran & Iran & M.E. $^{2}$ & Iran & Georgia & Georgia & Russia & Turkey & Turkey \\
\hline $\begin{array}{l}\text { Ch. Ass. } \mathbf{1}^{3} \\
\text { Cotoneaster nummularius } \\
\text { Lonicera nummulariifolia } \\
\text { Dactylis glomerata } \\
\text { Astragalus aegobromus } \\
\text { Asperula arvensis } \\
\text { Chalcanthus renifolius } \\
\text { Silene aucheriana } \\
\text { Silene marschalii } \\
\text { Astragalus citrinus }\end{array}$ & $\begin{array}{c}\text { V } \\
\text { V } \\
\text { V } \\
\text { IV } \\
\text { III } \\
\text { II } \\
\text { II } \\
\text { II } \\
\text { I }\end{array}$ & & & & & & & & & & \\
\hline $\begin{array}{l}\text { D. SubAss. } 2 \\
\text { Ajuga chamaecistus } \\
\text { Bupleurum exaltatum } \\
\text { Noaea mucronata } \\
\text { Astragalus podolobus } \\
\text { Johrenia platycarpa } \\
\text { Silene spergulifolia }\end{array}$ & I & $\begin{array}{l}\text { IV } \\
\text { IV } \\
\text { IV } \\
\text { III } \\
\text { III } \\
\text { III }\end{array}$ & & & & & & & & & \\
\hline $\begin{array}{l}\text { D. SubAss. } 3 \\
\text { Tanacetum polycephalum } \\
\text { Gypsophila aretioides } \\
\text { Pimpinella tragium } \\
\text { Helichrysum oligocephalum }\end{array}$ & & & $\begin{array}{l}\text { IV } \\
\text { III } \\
\text { III } \\
\text { II }\end{array}$ & & & & & & & & \\
\hline $\begin{array}{l}\text { D. SubAss. } 4 \\
\text { Cotoneaster kotschyi } \\
\text { Valerianella tuberculata } \\
\text { Pistacia atlantica }\end{array}$ & & & & $\begin{array}{l}\text { V } \\
\text { V } \\
\text { III }\end{array}$ & + & & & II & + & + & \\
\hline $\begin{array}{l}\text { Ch. Ass. 2-4 } \\
\text { Rhamnus pallasii } \\
\text { Ephedra major }\end{array}$ & & $\begin{array}{l}\text { V } \\
\text { III }\end{array}$ & $\begin{array}{l}\text { IV } \\
\text { IV }\end{array}$ & V & & $\begin{array}{l}\text { V } \\
\text { V }\end{array}$ & II & I & & & V \\
\hline $\begin{array}{l}\text { Ch. Order \& Class 1-5 } \\
\text { Cerasus microcarpa }\end{array}$ & IV & II & II & V & & & & I & & & \\
\hline
\end{tabular}




\begin{tabular}{|c|c|c|c|c|c|c|c|c|c|c|c|}
\hline Column No. & 1 & 2 & 3 & 4 & 5 & 6 & 7 & 8 & 9 & 10 & 11 \\
\hline Class & \multicolumn{5}{|c|}{ Junipero-Pistacietea Zohary 1973} & $\begin{array}{l}\text { Ephed- } \\
\text { retea }\end{array}$ & \multicolumn{2}{|c|}{ Not defined } & \multicolumn{3}{|c|}{$\begin{array}{l}\text { Quercetea pubescentis Kraft } 1955 \\
\text { (Tel et al., 2010) }\end{array}$} \\
\hline Order & \multicolumn{4}{|c|}{$\begin{array}{l}\text { Juniperetalia excelsae Ravanbakhsh \& } \\
\text { Hamzeh'ee } 2015\end{array}$} & 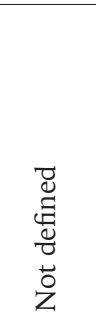 & 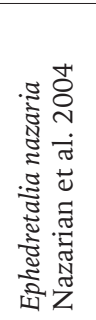 & 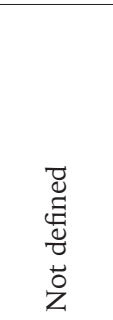 & 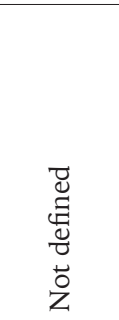 & 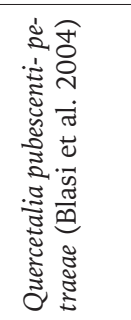 & 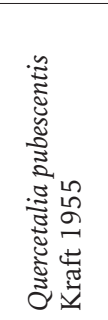 & 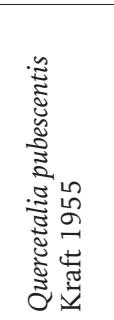 \\
\hline & \multirow{2}{*}{ 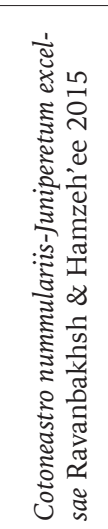 } & \multicolumn{3}{|c|}{$\begin{array}{l}\text { Rhamno pallasii-Junipere- } \\
\text { tum excelsae } \\
\text { Ravanbakhsh \& } \\
\text { Hamzeh'ee } 2015\end{array}$} & \multirow[b]{2}{*}{ 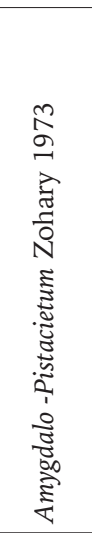 } & \multirow{2}{*}{ 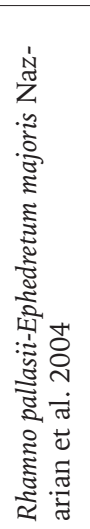 } & \multirow{2}{*}{ 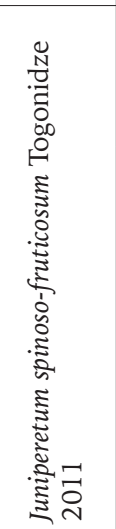 } & \multirow{2}{*}{ 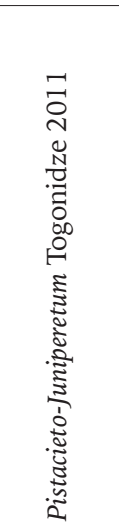 } & \multirow{2}{*}{ 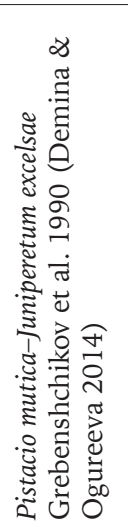 } & \multirow[b]{2}{*}{ 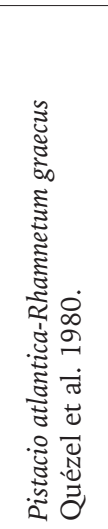 } & \multirow{2}{*}{ 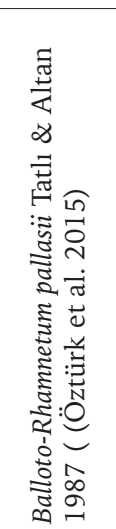 } \\
\hline $\begin{array}{l}\text { Association \& } \\
\text { Subassociation }\end{array}$ & & 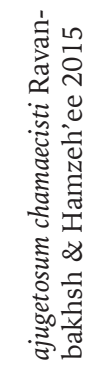 & 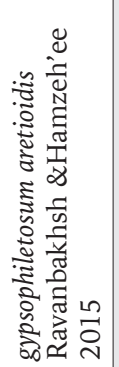 & 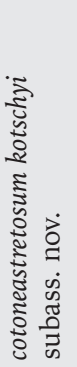 & & & & & & & \\
\hline Location & Iran & Iran & Iran & Iran & M.E. $^{2}$ & Iran & Georgia & Georgia & Russia & Turkey & Turkey \\
\hline Rubia florida & $\mathrm{V}$ & III & I & I & & & & & & & \\
\hline Berberis integerrima & $\mathrm{V}$ & IV & II & I & & & & & & & \\
\hline Conringia planisiliqua & II & II & III & II & & & & & & & \\
\hline Juniperus excelsa & $\mathrm{V}$ & $\mathrm{V}$ & $\mathrm{V}$ & III & & & II & & + & & \\
\hline Amygdalus lycioides & I & III & IV & $\mathrm{V}$ & + & & & & & & \\
\hline $\begin{array}{l}\text { Ch. Ass. 7-9 } \\
\text { Juniperus foetidissima } \\
\text { Juniperus oxycedrus } \\
\text { Paliurus spina-christi } \\
\text { Berberis iberica } \\
\text { Lonicera iberica } \\
\text { Spiraea hypericifolia } \\
\text { Ephedra procera } \\
\text { Jasminum fruticans } \\
\text { Cotoneaster integerrimus } \\
\text { Teucrium polium } \\
\text { Asparagus verticillatus }\end{array}$ & & III & IV & III & & & $\begin{array}{c}\text { III } \\
\text { II } \\
\text { III } \\
\text { I } \\
\text { I } \\
\text { II } \\
\text { I }\end{array}$ & $\begin{array}{l}\text { I } \\
\text { I } \\
\text { I }\end{array}$ & $\begin{array}{l}+ \\
+ \\
+\end{array}$ & & \\
\hline $\begin{array}{l}\text { Ch. Ass. } 10 \\
\text { Rhamnus graecus }\end{array}$ & & & & & & & & & & + & \\
\hline $\begin{array}{l}\text { Ch. Ass. } 11 \\
\text { Ballota nigra subsp. nigra } \\
\text { Polygonum convolvulus } \\
\text { Spiraea crenata } \\
\text { Acinos arvensis } \\
\text { Sobolewskia clavata }\end{array}$ & & & & & & & & & & & $\begin{array}{c}\text { V } \\
\text { V } \\
\text { IV } \\
\text { III } \\
\text { II }\end{array}$ \\
\hline $\begin{array}{l}\text { Ch. Order \& Class 9-11 } \\
\text { Continus coggyria } \\
\text { Teucrium chamaedrys } \\
\text { Quercus pubescens }\end{array}$ & & & & & & & & I & I & + & $\begin{array}{l}\mathrm{V} \\
\mathrm{V}\end{array}$ \\
\hline
\end{tabular}

${ }^{1}$ Ch: characteristic species; D: differential species; ${ }^{2}$ Middle East; ${ }^{3}$ It refers to the column numbers.

+ was applied when the constancy was not specified in the original article. 
Table 3. Eigenvalues and species-environment correlation coefficients for the first three components with the results of Monte Carlo test

\begin{tabular}{|c|c|c|c|}
\hline & $\begin{array}{c}\text { Com- } \\
\text { ponent } \\
1\end{array}$ & $\begin{array}{c}\text { Com- } \\
\text { ponent } \\
2\end{array}$ & $\begin{array}{c}\text { Com- } \\
\text { ponent } \\
3\end{array}$ \\
\hline Eigenvalue & $0.36^{* *}$ & $0.31^{* *}$ & $0.23^{* *}$ \\
\hline \multicolumn{4}{|l|}{$\begin{array}{l}\text { Cumulative percentage variance } \\
\text { explained: }\end{array}$} \\
\hline of species data & 12.2 & 22.3 & 30 \\
\hline of species-environment relation & 23.2 & 42.6 & 57.2 \\
\hline Pearson correlation & $0.95^{* *}$ & $0.95^{*}$ & 0.95 \\
\hline Kendall (Rank) correlation & $0.80^{* *}$ & $0.83^{*}$ & 0.69 \\
\hline
\end{tabular}

"Significant at $\mathrm{P}<0.01$ level; " Significant at $\mathrm{P}<0.05$ level.
Table 4. The correlation of environmental variables with first three components of CCA

\begin{tabular}{lccc}
\hline \multirow{2}{*}{ Variables } & \multicolumn{3}{c}{ Correlations $^{1}$} \\
\cline { 2 - 4 } & Component 1 & Component 2 & Component 3 \\
\hline Altitude & 0.863 & 0.361 & 0.186 \\
Aspect & 0.264 & 0.168 & -0.422 \\
Slope & -0.210 & 0.544 & -0.618 \\
pH & 0.208 & -0.607 & 0.262 \\
Lime & -0.118 & -0.833 & -0.108 \\
Total N & 0.640 & -0.103 & 0.123 \\
Organic C & 0.664 & -0.153 & 0.005 \\
SP $^{2}$ & 0.644 & -0.377 & 0.162 \\
Sand & -0.251 & 0.498 & -0.484 \\
Clay & 0.055 & -0.432 & 0.261 \\
\hline
\end{tabular}

${ }^{1}$ Intraset correlations of ter Braak (1986); ${ }^{2}$ Water saturation [\%]

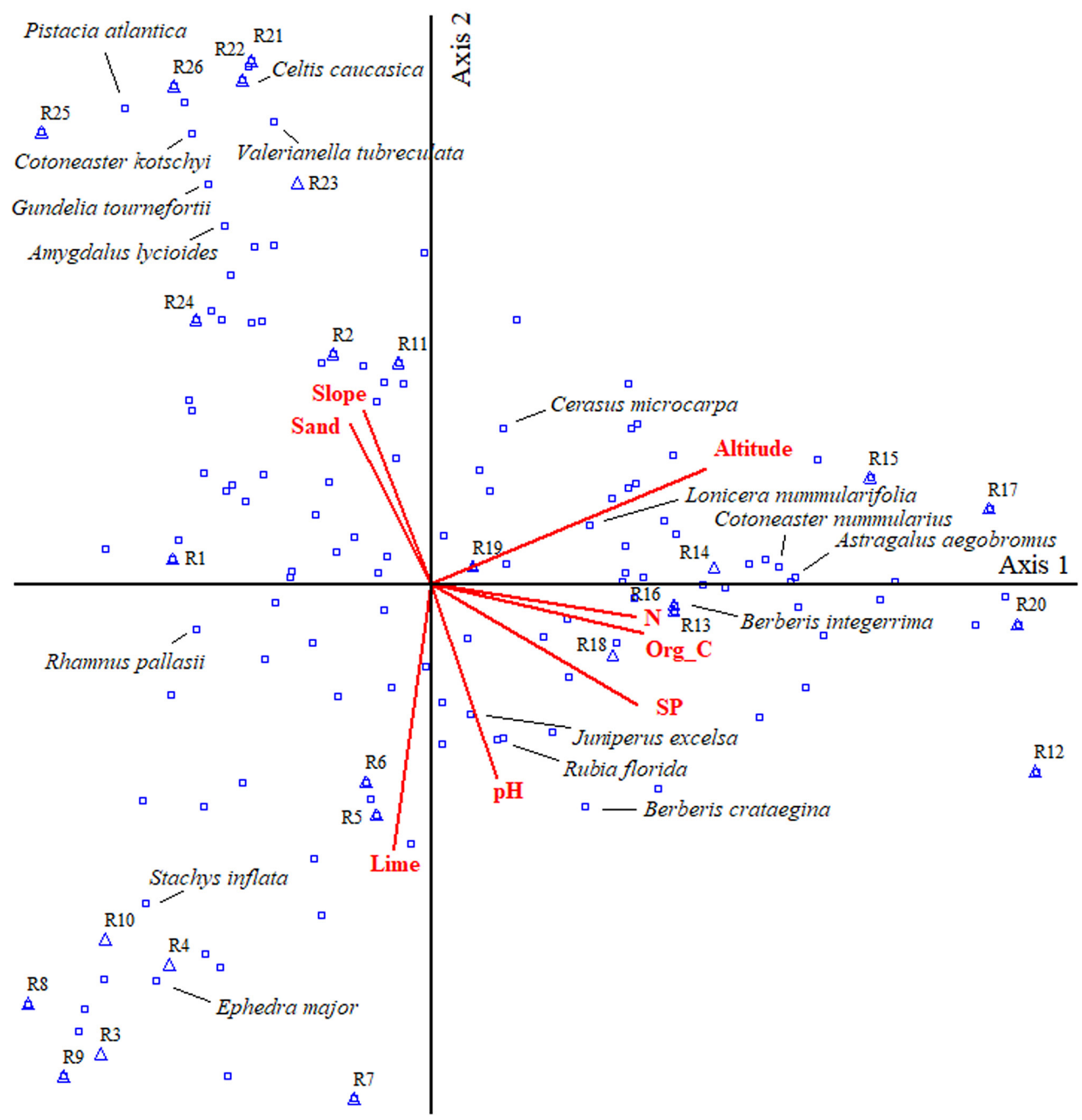

Fig. 3. The distribution of species and plots in the CCA ordination diagram (axis 1 and axis 2)

The arrows for environmental variables indicate the direction of maximum change of that variable; $\Delta$ - relevés with their names (R1, R2, etc.); $\square$ species (just diagnostic and highly-correlated species are presented). 
Table 5. The average of environmental variables for different groups using Duncan mean comparison ${ }^{1}$

\begin{tabular}{lcccccc}
\hline \multicolumn{1}{c}{ Variable } & Rh. pallasii-J. excelsae & SD $^{2}$ & C. nummulariis-J. excelsae & SD & Cotoneastretosum kotschyi & SD \\
\hline Altitude (m) & $2216^{\mathrm{a}}$ & $(101)$ & $2488^{\mathrm{b}}$ & $(137)$ & $2333^{\mathrm{c}}$ & $(32)$ \\
Slope \% & $38.5^{\mathrm{a}}$ & $(14.3)$ & $43.5^{\mathrm{a}}$ & $(10.8)$ & $61.7^{\mathrm{b}}$ & $(6.8)$ \\
Aspect & $162^{\mathrm{a}}$ & $(40)$ & $189^{\mathrm{a}}$ & $(51)$ & $181^{\mathrm{a}}$ & $(36)$ \\
$\mathrm{pH}$ & $7.86^{\mathrm{a}}$ & $(0.28)$ & $7.78^{\mathrm{a}}$ & $(0.13)$ & $7.42^{\mathrm{b}}$ & $(0.19)$ \\
Lime \% & $21.3^{\mathrm{a}}$ & $(8.9)$ & $12.3^{\mathrm{b}}$ & $(7.0)$ & $4.2^{\mathrm{c}}$ & $(2.3)$ \\
Organic matter \% & $2.59^{\mathrm{a}}$ & $(1.16)$ & $5.02^{\mathrm{b}}$ & $(2.66)$ & $1.87^{\mathrm{a}}$ & $(0.72)$ \\
$\mathrm{N} \%$ & $0.12^{\mathrm{a}}$ & $(0.05)$ & $0.20^{\mathrm{b}}$ & $(0.10)$ & $0.10^{\mathrm{a}}$ & $(0.06)$ \\
SP \% & $42.3^{\mathrm{a}}$ & $(4.5)$ & $48.2^{\mathrm{a}}$ & $(8.7)$ & $33.6^{\mathrm{b}}$ & $(8.8)$ \\
Sand \% & $39.29^{\mathrm{a}}$ & $(13.51)$ & $40.20^{\mathrm{a}}$ & $(13.73)$ & $60.99^{\mathrm{b}}$ & $(12.48)$ \\
Silt \% & $33.01^{\mathrm{a}}$ & $(9.11)$ & $33.50^{\mathrm{a}}$ & $(9.37)$ & $22.11^{\mathrm{b}}$ & $(7.91)$ \\
Clay \% & $27.69^{\mathrm{a}}$ & $(7.19)$ & $25.30^{\mathrm{ab}}$ & $(10.27)$ & $17.91^{\mathrm{b}}$ & $(8.12)$ \\
\hline
\end{tabular}

${ }^{1}$ Similar letters indicate no significant difference amongst groups; ${ }^{2}$ Standard Deviation.

nitrogen and organic matter content. Similarly, the relevés of Rh. Pallasii-J. excelsae are located along the negative direction of first axis. Ephedra major, Stachys inflata and Teucrium polium L. showing a tendency to grow in more lime-rich habitats with low organic matter.

\section{Analysis of variance and comparison of environmental variables in the vegetation groups}

The ANOVA results showed that altitude, slope, $\mathrm{pH}$, lime, organic matter, soil nitrogen, water saturation level, sand and silt content are significantly different amongst vegetation groups (Table 5). In terms of altitude, cotoneastretosum kotschyi is located between C. nummulariis-J. excelsae and Rh. pallasii-J. excelsae (Table 5). The subassociation cotoneastretosum kotschyi is distributed on average at $60 \%$ slopes which is significantly higher than those for C. nummulariis-J. excelsae or Rh. pallasii-J. excelsae. The soil pH and lime, organic carbon and nitrogen, water saturation percent are lower in cotoneastretosum kotschyi compared to two other groups. In terms of soil texture, cotoneastretosum kotschyi also contains higher amounts of sand, and lower amounts of silt and clay compared to the two other groups, with a significant difference observed between sand and silt (Table 5). Furthermore, the organic matter and water saturation level in C. nummulariis-J. excelsae is significantly higher than in Rh. pallasii-J. excelsae, and the first community is located at higher altitude compared to the second community.

\section{Discussion}

\section{Vegetation}

The genus Cotoneaster is considered an important taxon in woodlands of Irano-Turanian, Caucasian and Mediterranean regions (i.e. Cotoneaster nummularius in Querco vulcanicae-Juniperetum excelsae in Turkey (Kargioglu \& Tatli, 2005; Ozkan et al., 2010) and in Cotoneastro nummulariis-Juniperetum excelsae in Iran (Ravanbakhsh et al., 2016); C. integerrimus Medik. as a characteristic species of Pino-Juniperetea Rivas-Martinez 1964 in the Central and Eastern Mediterranean region (Brullo et al., 2001) and in Pistacieto-Juniperetum of Georgia (Togonidze, 2011) and C. racemiflora in Juniper forests of the Himalayas (Ahmed, 2006). Based on the results of this study, the Cotoneaster shrublands of the Alborz are placed in Cotoneastro nummulariis-Juniperetum excelsae and Rhamno pallasii-Juniperetum excelsae cotoneastretosum kotschyi subass. nov. Introduction of the new subassociation increased the number of subassociations of Rhamno pallasii-Juniperetum excelsae to three (Table 2). The subassociations already introduced, gypsophiletosum aretioidis and ajugetosum chamaecisti, were restricted to the rocky habitat and the mountains to dry plains, respectively (Ravanbakhsh et al., 2016), while cotoneastretosum kotschyi is located in the sandy-loam soil and mountainous areas far from the dry plains. According to the literature, different species of Cotoneaster appear with Rhamnus species in different syntaxa together and sometimes with Juniperus (Kaya, 1999; Abido \& Kurbaisa, 2003; Togonidze, 2011) which reflects the phytosociological relationship amongst these genera. In our study area, these genera were presented together as well, and C. kotschyi, Rh. pallasii and J. excelsa along with other species which constitute a unique vegetation unit with special floristic composition.

\section{Environmental variables}

Among the environmental factors, slope, soil texture, $\mathrm{pH}$, lime and saturation level were the most important distinguishing factors of cotoneastretosum kotschyi. Moreover, this community appears in the altitudes of 2,200-2,430 $\mathrm{m}$ a.s.1., i.e. in an intermediate height range between the $C$. nummulariis-J. excelsae and Rh. pallasii-J. excelsae. The species composition of 
cotoneastretosum kotschyi also showed the characteristic species of both mentioned communities such Rhamnus pallasii and Cotoneaster nummularius.

Soil organic matter and nitrogen were the distinctive factors between the two communities of $C$. nummulariis-J. excelsae and Rh. pallasii-J. excelsae. Generally $R h$. pallasii-J. excelsae can be more frequently observed in shallow, unqualified soil compared to the other community. In Juniper woodlands in East Georgia the Rhamnus pallasii can also be seen as an understory shrub in rocky shallow soils (Togonidze, 2011). In North Central Alborz (in Elika ecoton) Rhamno pallasii-Ephedretum majoris was observed on calcareous soils and at warmer south geographical aspects (Nazarian et al., 2004). Furthermore, Rhamnus rhodopeus was reported as the differential species of alliance in habitats of Querco trojanae-Juniperetum excelsae in shallow soil with limestone bedrock, on the southern slopes in Macedonia (Matevski et al., 2010), which is similar to Rhamno pallasii-Juniperetum excelsae habitat in Alborz mountains of Iran. On the other hand, Cotoneaster nummularius is the characteristic species of Juniper communities on brown forest soils with limestone bedrock, and 5-20\% slope (Kargioglu \& Tatli, 2005) indicating preference or a deeper soil and more favourable conditions compared to Rh. pallasii-J. excelsae habitat.

The main differences of the cotoneastretosum kotschyi habitat with its superior association (i.e. $R h$. pallasii-J. excelsae) are in soil texture, $\mathrm{pH}$, lime and slope, so that cotoneastretosum kotschyi was distributed in more acidic soils, with lower lime, lighter textures (i.e. sandy-loam) and steeper slopes.

The Cotoneaster genus have inherent ecological diversity. For instance, Cotoneaster integerrimus Medik. grows on rocky habitats with low or medium soil depth in Georgia (Togonidze, 2011) and on lithosols and entisols, which are typically poor in humus and organic matter in the Central and East Mediterranean regions (Brullo et al., 2001), while Cotoneaster nummularius was observed in low-slope habitats with brown forest soils in Turkey (Kargioglu \& Tatli, 2005). Cotoneaster racemiflora was reported in Juniper communities in the Himalayas at altitude of 2,100-2,800 m a.s.l. (Ahmed, 2006). Therefore, different Cotoneaster species can occur in varying environmental conditions, ranging from moist habitats with fertile soils to those with shallow and poor soils. These conditions can also be seen in Alborz, where Cotoneaster nummularius is the indicator of communities with relatively evolved soils. However, Cotoneaster kotschyi appears in habitats featuring poor and shallow soils. In terms of the ecological nature, the latter species is in fact similar to Rhamnus pallasii, the characteristic species of Juniperus communities on shallow soils and stony lands. This was confirmed by both phytosociological and environmental variables analysis. Therefore, locating the syntaxon with the diagnostic species of Cotoneaster kotschyi within Rhamno pallasii-Juniperetum excelsae is ecologically confirmed.

The floristic composition of Juniperus excelsa group in the Shohada protected area in western Azerbaijan with species such as Amygdalus pabotii Browicz, Rhamnus pallasii and Pistacia atlantica (Hassanzadeh \& Mohammdi, 2010) is similar to those that were studied in the Alborz region. This habitat can be observed in altitude of 1,650-2,200 m a.s.l., slopes $>60 \%, \mathrm{pH}$ of 8 and sandy clay in texture, which are ecologically similar to our study area.

Co-occurring plant species of a targeted species can be used to define suitable habitats, taking into account biotic interactions (Baumberger et al., 2012). Regarding the results of this study, Rooteh Forest Reserve has a unique plant composition (in particular in terms of tree and shrub species), thus it must be protected as genetic reserve and seed bank for the further studies as well as for rehabilitation of the C. kotschyi habitat.

\section{References}

Abido MS \& Kurbaisa MS (2003) The present status of the Syrian Juniper forests on the East Lebanon Mountain Chain, Syrian Arab Republic. Arab Gulf Journal of Scientific Research 21: 64-70.

Ahmed M, Husain T, Sheikh AH, Hussain SS \& Siddiqui MF (2006) Phytosociology and structure of Himalayan forests from different climatic zones of Pakistan. Pakistan Journal of Botany 38: 361-383.

Assadi M (1988-2016) Flora of Iran. No. 1-85. Research Institute of Forests and Rangelands, Tehran, Iran.

Baumberger T, Croze T, Affre L \& Mesléard F (2012) Co-occurring species indicate habitats of the rare Limonium girardianum. Plant Ecology and Evolution 145: 31-37. doi:10.5091/plecevo.2012.685.

Beals EW (1965) The remnant cedar forests of Lebanon. Journal of Ecology 53: 679-694.

Biondi E (2011) Phytosociology today: Methodological and conceptual evolution. Plant Biosystems 145: 19-29. doi:10.1080/11263504.2011.60274 8.

Blasi C, Pietro R Di \& Filesi L (2004) Syntaxonomical revision of Quercetalia pubescenti-petraeae in the Italian Peninsula. Fitosociologia 41: 87-164.

Boratyński A, Kosiński P, Kwiatkowski P, Szczęśniak E \& Świerkosz K (1999) Protected and deserving protection trees and shrubs of the Polish Sudety Mts. with their prealps. 11. Cotoneaster integerrimus Medicus i C. niger (Thunb.) Fr. Arboretum Kórnickie 44: 5-22.

Braun-Blanquet J (1951) Pflanzensoziologie, grundzüge der vegetationskunde. Springer, Wien. 
Brullo S, Giusso Dell Galdo G \& Guarino R (2001) The orophilous communities of the Pino-Juniperetea class in the Central and Eastern Mediterranean area. Feddes Repertorium 112: 261-308. doi:10.1002/fedr.20011120308.

Chytrý M \& Tichý L (2003) Diagnostic, constant and dominant species of vegetation classes and alliances of the Czech Republic: a statistical revision. Vol. 108. Masaryk University, Brno, Czech Republic.

Chytrý M, Tichý L, Holt J \& Botta-Dukát Z (2002) Determination of diagnostic species with statistical fidelity measures. Journal of Vegetation Science 13: 79-90. doi:10.1111/j.1654-1103.2002. tb02025.x.

Demina ON \& Ogureeva GN (2014) The preservation of the relic xerophytic sub-mediterranean communities in the southern Russia. European vegetation survey, 23rd International Workshop of the European Vegetation Survey, pp. 62-63.

Elzein TM, Blarquez O, Gauthier O \& Carcaillet C (2011) Allometric equations for biomass assessment of subalpine dwarf shrubs. Alpine Botany 121: 129-134. doi:10.1007/s00035-011-0095-3.

Hassanzadeh A \& Mohamadi LM (2010) Plant community traits of Shohada Protected Area, West Azerbijan, Iran. Notulae Scientia Biologicae 2: 29-35. doi:10.15835/nsb.2.1.3566.

Hill MO (1979) TWINSPAN: A FORTRAN program for arranging multivariate data in an ordered twoway table by classification of the individuals and attributes. Cornell Ecology Programs Series. Cornell University, Ithaca, NY.

IUCN (2017) The IUCN Red List of Threatened Species. Version 2017-1. http://www.iucnredlist.org.

Kartoolinejad D \& Moshki A (2014) Changes in Juniperus polycarpos community in response to physiographical factors (Hezarmasjed Mountain, Iran). Austrian Journal of Forest Science 131: 215-232.

Kargioglu M \& Tatli A (2005) A phytosociological research on the forest vegetation of Yandag (Isparta-Turkey). Pakistan Journal of Biology Science 8: 929-939. doi:10.3923/pjbs.2005.929.939.

Kaya Y (1999) Furat Vadisi'nde erozyon ve erozyon alanında iyi gelișen bitkiler. Turkish Journal of Agriculture \& Forestry 23: 7-24.

Kent M \& Coker P (1994) Vegetation description and analysis: a practical approach. Wiley-Blackwell.

Khatamsaz M (1992) Flora of Iran no. 6: Rosaceae (ed. by M Assadi) Research Institute of Forests and Rangelands, Tehran, Iran, pp. 209-239.

Klein JC (2001) La végétation altitudinale de l'Alborz Central (Iran). Institut Français de Recherche en Iran, Peeters, Louvain, France.

Kraft HD (1955) Over de invloed van de mens op de plantengroei. De Levende Natuur 58: 93-99.
Lepš J \& Šmilauer P (1999) Multivariate analysis of ecological data. Faculty of Biological Sciences. University of South Bohemia, České Budějovice, Czech Republic.

McCune B \& Mefford MJ (1999) PC-ORD: multivariate analysis of ecological data. Version 4. MjM Software Design, Gleneden Beach, Oregon, USA.

Marvie Mohadjer MR (2005) Silviculture. University of Tehran, Tehran, Iran.

Matevski V, Čarni A, Kostadinovski M, Marinšek A, Mucina L, Paušič A \& Šilc U (2010) Notes on phytosociology of Juniperus excelsa in Macedonia (southern Balkan Peninsula). Hacquetia 9: 161165. doi:10.2478/v10028-010-0005-z.

Mohammadi N, Sharififar F, Koohpayeh A \& Daneshpajouh M (2015) Traditional and Ethnobotanical uses of medicinal plants by ancient populations in Khabr and Rouchon of Iran. Journal of Applied Pharmaceutical Science 5: 101-107. doi:10.7324/ JAPS.2015.501117.

Nazarian H, Ghahreman A, Atri M \& Assadi M (2004) Ecological factors affecting parts of vegetation in north Iran (Elika and Duna Watersheds) by employing eco-phytosociological method. Pakistan Journal of Botany 36: 41-64.

Ozkan K, Gulsoy S, Aerts R \& Muys B (2010) Site properties for Crimean juniper (Juniperus excelsa) in semi-natural forests of south western Anatolia, Turkey. Journal of Environmental Biology 31: 97-100.

Öztürk M, Özçelik H\& Behçet L (2015) General characteristics of flora and vegetation formations of eastern Anatolia Region and its environs (Türkiye). SDÜ Journal of Science 10: 23-48.

Palmer MW (1993) Putting things in even better order: the advantages of canonical correspondence analysis. Ecology 74: 2215-2230.

Poore MED (1955) The use of phytosociological methods in ecological investigations: I. The Braun-Blanquet system. Journal of Ecology 43: 226-244. doi:10.2307/2257132.

Quézel P, Barbero M \& Akman Y (1980) The forest vegetation of northern Anatolia. Phytocoenologia 8: 365-519.

Ravanbakhsh H, Hamzeh'ee B, Etemad V, Marvie Mohadjer MR \& Assadi M (2016) Phytosociology of Juniperus excelsa M. Bieb. Forests in Alborz mountain range in the north of Iran. Plant Biosystems 150: 987-1000. doi:10.1080/11263504.201 4.1000420 .

Ravanbakhsh H, Marvie Mohadjer MR, Zahedi G \& Shirvani A (2010) Forest typology in relation with altitude gradient on southern slopes of central Alborz Mountains (Latian Dam Watershed). Journal of Forest and Wood Products 64: 9-22.

Rechinger KH (1963-2005) Flora Iranica. No. 1-176. Akademische Druck, Graz, Austria. 
Ried H (1969) Flora Iranica. No. 66: Rosaceae I (ed. by $\mathrm{KH}$ Rechinger) Akademische Druck, Graz, Austria, pp. 14-26.

Sagheb-Talebi Kh, Sajedi T \& Pourhashemi M (2014) Forests of Iran: A treasure from the past, a hope for the future. Plant and Vegetation 10. Springer, Dordrecht. doi:10.1007/978-94-007-7371-4.

Tel AZ, Tatli A \& Varol O (2010) Phytosociological structure of Nemrut Mountain (Adiyaman/Turkey). Turkish Journal of Botany 34: 417-434.

ter Braak CJF (1986) Canonical correspondence analysis: a new eigenvector technique for multivariate direct gradient analysis. Ecology 67: 1167-1179.

ter Braak CJF \& Smilauer P (2002) CANOCO reference manual and CanoDraw for Windows user's guide: software for canonical community ordination (version 4.5). www. canoco. Com.

Tichý L (2002) JUICE, software for vegetation classification. Journal of Vegetation Science 13: 451453. doi:10.1111/j.1654-1103.2002.tb02069.x.

Togonidze P (2011) Typology of East Georgian open juniper woodlands. Bulletin of the Georgian National Academy of Sciences 5: 103-106.

Weber HE, Moravec J \& Theurillat JP (2000) International code of phytosociological nomenclature. 3rd edition. Journal of Vegetation Science 11: 739-768. doi:10.2307/3236580.

Zohary M (1973) Geobotanical foundations of the Middle East. G. Fischer, Stuttgart, Germany. 